\title{
Géneros periodísticos de opinión: análisis y recuperación de información factual en sistemas documentales
}

\author{
Antonio GARCíA GUTIÉRREZ \\ Universidad de Sevilla \\ algarcia@us.es
}

Recibido: 4 de septiembre de 2013

Aceptado: 13 de enero de 2014

\begin{abstract}
Resumen
Los géneros de opinión no solo ocupan un gran espacio en la prensa escrita sino también en los archivos digitales mediáticos. Al igual que otros géneros periodísticos, previamente investigados desde la óptica documental, los textos de opinión son tradicionalmente analizados en los sistemas digitales mediante técnicas documentales convencionales que no permiten responder con datos factuales (asuntos, argumentos, valoraciones, causas o fines del análisis, conclusiones o propuestas...) a preguntas pronominales de los usuarios (¿quién, por qué, cómo o qué... valora, analiza o concluye?). Se propone, como parte de un amplio programa de investigación que abarca todos los géneros periodísticos, atendiendo a sus tipologías y estrategias discursivas, un procedimiento adhoc para la organización y obtención, en sistemas digitales, de información factual de los textos de opinión mediante categorías homologables y testadas en otros géneros.
\end{abstract}

Palabras clave: Documentación periodística, Géneros periodísticos de persuasión, Textos de opinión, Información factual, Búsqueda pronominal

\section{Opinion Texts in Journalism: Analysis and Retrieval of Factual Data in Information Systems}

\begin{abstract}
Opinion texts not only occupy a large space throughout press pages, but also in the digital media archives. As other journalistic genres, previously studied from the information systems perspective, opinion texts are traditionally analyzed in such systems using conventional techniques of extraction of metadata, operations that do not allow factual data (issues discussed, evaluations, causes or purposes of the analysis, conclusions and proposals...) as an answer to pronominal questions asked by users (who, why, how or what... assesses, analyses or proposes?). As part of a broad program of research addressing all the journalistic genres and taking into account its typologies and discursive strategies, an adhoc procedure for the organization and retrieval of factual data from opinion texts is proposed keeping the genuine nature and potentials of this particular genre.
\end{abstract}

Keywords: Journalistic Documentation, Persuasion Genres, Opinion Texts, Factual Data, Pronominal Search

\section{Referencia normalizada}

GARCÍA GUTIÉRREZ, Antonio (2014): “Géneros periodísticos de opinión: análisis y recuperación de información factual en sistemas documentales”. Estudios sobre el Mensaje Periodístico. Vol. 20, Núm. 1 (enero-junio), págs.: 377-394. Madrid, Servicio de Publicaciones de la Universidad Complutense.

Sumario: 1. Introducción. 2. Hipótesis. 3. Textos de opinión: estructura, retórica y acotaciones desde la óptica documental. 4. Metodología y aparato teórico conceptual. 5. Corpus, observables y construcción de macroproposiciones; 5.1. Sobre autores y agentes; 5.2. Categorías de interrogación pronominal; 5.3. Acciones; 5.4. Conectores y ordenación lógico-sintáctica de MPs. 6. Modelo de interrogación pronominal de textos de opinión. 7. Conclusiones. 8. Referencias bibliográficas. 


\section{Introducción}

Los textos pertenecientes a los llamados géneros periodísticos de opinión ocupan un amplio espacio físico en los media, incluso en los tradicionalmente dominados por el periodismo informativo. A pesar de ser cada vez más compleja la disyunción entre hechos y opiniones, incluso para algunos constructivistas radicales como Bruno Latour "no solo serían construcciones las explicaciones de los hechos sino también los hechos mismos" (Latour y Woolgar, 1995), entenderemos por textos de opinión, a partir de un conocimiento empírico ordinario, aquellos que explicitan la posición del autor (y no solo mediante su firma) y que adoptan los cánones estructurales y las estrategias discursivas de persuasión propias de estos géneros periodísticos ( $c f r$. Santamaría y Casals, 2000). No es posible adoptar una tipología universal e inmutable de estos géneros, habida cuenta de los crecientes procesos de hibridación mediática, y tampoco es objetivo ni una necesidad de este trabajo. Por lo tanto, nos apoyaremos en las denominaciones y clasificaciones dominantes que, desde un punto de vista práctico, circulan por los medios y en los trabajos clásicos y estándares en la materia (Martínez Albertos, 2000; Gomis, 2008)

Por otra parte, la proliferación de textos de opinión en las bases y sistemas de documentación periodística viene a incrementar los niveles de ruido (extracción de datos irrelevantes o no deseados) y silencio (datos relevantes no recuperables) ya generados en las memorias mediáticas por millones de noticias, reportajes y entrevistas cuyo tratamiento documental hemos desarrollado en otro lugar (2013). Aquellas investigaciones abordaban la construcción de metodologías pragmatista de análisis de noticias, entrevistas y textos periodísticos declarativos desde una perspectiva basada en el comportamiento y necesidades del usuario que busca datos puntuales o "factuales" a través de las estructuras conceptuales de los mismos. En los últimos decenios, el grueso de la investigación sobre análisis documental periodístico se ha focalizado, no obstante, bien en la obtención de resúmenes, metadatos o referencias, bien en lenguajes y reglas de representación soslayando la exploración de otras vías de análisis y búsqueda de información susceptibles de aportar soluciones compatibles con los productos documentales clásicos de los servicios de documentación mediáticos.

A pesar del modelo de interrogación y organización textual propuesto sobre algunos géneros informativos, en sus conclusiones también se subrayaba la necesidad de realizar una aproximación diferente del procedimiento pronominal aludido al aplicarlo sobre textos de idiosincrasia y formalismos discursivos también diferentes como ocurre con los géneros de opinión pero buscando su compatibilidad en sistemas integrados de información.

Es indiscutible que los usuarios -periodistas, editores, realizadores e incluso historiadores o público general- no solo buscan textos íntegros o referencias en los sistemas masivos de información digital relacionados con hechos y descripciones (géneros informativos), sino también datos puntuales articulados a opiniones, sesgos y posiciones de determinados medios o autores (géneros de opinión, también llamados argumentativos o persuasivos). A la hemeroteca digital de un medio se le solicitan argumentos o conclusiones específicos sobre un asunto de actualidad que el propio medio hace públicos (no todo el texto del editorial sino un dato específico) o elabo- 
rados por diferentes colaboradores o productores de la opinión (analistas, críticos, columnistas, etc.) cuya visión o posición personal es, desde esta óptica, tan o más importante para el usuario que el propio hecho comentado. Tal imperativo es justificación suficiente para dedicar a los textos periodísticos de opinión un estudio específico.

Los resultados de esta investigación, todavía de orden lógico-semántico y cuya meta a medio plazo es la gestión automática de buena parte de la organización pronominal de textos periodísticos en castellano (compatible con otros modos analíticos convencionales), deben ser encuadrados a corto plazo en la construcción gradual de una aplicación inteligente llevada a cabo junto a investigadores del área de sistemas y lenguajes informáticos pero, además, pueden ser utilizados como procedimiento de mayor objetivación del análisis de documentos periodísticos (sometido habitualmente a criterios no parametrizables como la intuición, la experiencia o el "sentido común" de los documentalistas de medios) por parte de profesores y programas de formación en documentación periodística con vistas a una redefinición de los roles futuros del documentalista de prensa.

\section{Hipótesis}

Partiendo de la evidencia de que los usuarios necesitan que los sistema documentales les proporcionen datos puntuales y precisos de los textos periodísticos de opinión, la hipótesis que desbrozaremos es la siguiente: "es posible organizar y extraer información factual de textos periodísticos de opinión mediante categorías de interrogación pronominal que giran en torno a un núcleo reducido de acciones, conectores y predicados organizados en macroproposiciones. Dichas categorías conceptuales se derivan de una aplicación pragmática de la teoría de casos universales desarrollada en torno a un reducido grupo de acciones controladas y externas a los contenidos abordados que es vinculable con una macroestructura estable -exposición-argumentación-conclusiónen los géneros de opinión convencionales. A diferencia del protocolo de análisis de géneros informativos (un enunciado por macroproposición), las macroproposiciones de los textos de opinión pueden necesitar en ocasiones más de un enunciado para atender variables discursivas derivadas de su macroestructura y plasmadas en dichos textos".

\section{Textos de opinión: estructura, retórica y acotaciones desde la óptica docu- mental}

Es obvia la dificultad de normalizar un protocolo de procedimiento de análisis documental para textos de la heterogeneidad conceptual y estructural de los textos de opinión, acogidos bajo un mismo género por los manuales cuyo objetivo es la persuasión. En relación a su tipología, estructura y funciones remitimos al exhaustivo trabajo de Santamaría y Casals (2000) y a una actualización en la investigación colectiva de Sánchez-Calero et al. (2011) por su tratamiento más específico del rol de la argumentación en estos géneros y los procesos de mestizaje tipológico que favorecen los medios y técnicas digitales. Los parámetros recogidos de las citadas obras, recortados por nuestros objetivos (un modelo de interrogación pronominal de textos de opinión), han sido clarificadores para el modo de abordaje y las soluciones específicas que se apor- 
tan desde la perspectiva de búsquedas pronominales de estos textos en bases periodísticas generales, en las que conviven con otros muchos géneros y producciones (fotografías, vídeos, etc.), si bien hubieron de ser hechas ciertas acotaciones imprescindibles establecidas en el presente epígrafe.

La dificultad de normalización de tanta diversidad temática, estilística, tipológica...decíamos que es obvia, pero también es evidente la búsqueda masiva y creciente de este tipo de textos en las bases de datos periodísticas, necesidad que justifica una investigación documental que, en primera instancia, inicie el abordaje estructuralmente, mitigándose de este modo la incidencia de las particularidades temáticas y de estilo de cada texto en un procedimiento documental que debe ser común, a pesar de que los matices serán cautelosamente tratados en el procedimiento o aislados y relegados, en caso de irresolubilidad inmediata, a investigaciones ulteriores.

Los textos de opinión presentan, desde la óptica de su sistematización documental y con independencia del medio de publicación o de sus autores, las siguientes propiedades compartidas:

a) extensión breve impuesta por el medio, que no suele superar la decena de párrafos, lo que los hace manejables desde la óptica de su desestructuración documental mediante mecanismos lógico-semánticos, como fase previa a una automatización del procedimiento, a diferencia de ensayos y otros géneros críticos no periodísticos de extensión y complejidad superiores.

b) desvelamiento claro de la posición del autor individual o institucional (en editoriales) mediante argumentos, conclusiones y valoraciones positivas, negativas o explícitamente (y estratégicamente) neutras a diferencia de noticias y otros géneros informativos en las que las posiciones están más veladas y tácitas cuando no negadas por una pretendida objetividad. Esta intencionalidad de desvelamiento de la opinión determina el tipo de estructura discursiva dominante en estos géneros frente a las configuraciones formales de otros.

c) retórica ilimitada, mediante un uso frecuente de figuras (rayando lo literario en ciertas producciones y autores), y estrategias discursivas de persuasión, lo que dificulta el análisis automático y hace necesaria una "traducción" a lenguaje descriptivo. Entre los diversos géneros de opinión, no obstante, hay grandes diferencias respecto a los recursos argumentativos. En general transcurren de los casos más sobrios y cercanos al silogismo en editoriales y análisis a una mayor carga retórica y hasta "psicagógica" (técnica de persuasión -ya tratada por Aristóteles- que apela a las emociones más que a la razón, muy presente en el discurso publicitario) cuando no abiertamente erística (técnica dialéctica que busca "llevarse" a toda costa la razón (vencer al oponente) y no convencer argumentalmente que se tiene, como ocurre habitualmente en el debate político ( $c f r$. Schopenhauer, 1996)) trasladado a columnas y críticas de calidad literaria y orografía argumental a la medida de la personalidad de sus autores.

d) articulación de la totalidad del texto respecto a la posición del autor o medio y no respecto los hechos como, pretendidamente, ocurre con las noticias. Tal subordinación abre la posibilidad de organizar, analizar y representar la ilimitada diferencia conceptual, temática y retórica de los textos e incluso la limitada diferencia estructural y extensional, en función de contadas acciones derivadas de opinar, parte de la hi- 
pótesis que permite un inicial abordaje de la heterogeneidad bajo un protocolo documental controlado.

e) una macroestructura canónica (Van-Dijk, 2001) con mayor grado de explicitación, ordenación y extensión basada en la exposición breve de un asunto (que se da por conocido), argumentos para sostener la posición del autor o medio respecto al asunto y, en su caso, relación de conclusiones, advertencias o propuestas. Si bien ésta sería la macroestructura general de un prototipo teórico de texto de opinión, los textos empíricos que encontramos la camuflan bajo un mar de formalismos y retóricas al servicio de la persuasión, por lo que algunos elementos macroestructurales pueden estar muy desarrollados, insinuados o totalmente omitidos, además de aparecer en cualquier orden. Por ejemplo, las conclusiones o propuestas que en la investigación científica han de aparecer separadas de la argumentación, en los textos de opinión pueden estar mezcladas con los argumentos cuando no implícitas, vagamente deslizadas o relegadas a un futuro en el que se disponga de datos suficientes para hacer un dictamen, lo que sitúa al documentalista ante decisiones analíticas complicadas, fragmentadas, serializadas, pues sus límites operacionales se "deben" a lo explicitado en un texto temporalmente clausurado y no en un ilimitado relato, si bien con conciencia del mismo.

Realizando la observación de textos de opinión desde la óptica de su organización temático-documental, se infiere que es posible tipificarlos, con independencia de su género específico, en temáticamente unitarios, segmentados y fragmentados. En el primer tipo, la argumentación gira en torno a un tema específico o macrotema, en su caso dividido en partes o casos inseparables de su macro. En los textos segmentados, se identifican dos o más temas suficientemente desarrollados, esto es, con autonomía significativa propia. Los texto fragmentados, por último, atomizan los comentarios microtemáticamente siendo, en realidad, más relevante el lenguaje personal del articulista que el hecho o sujeto comentados (por ejemplo, F. Umbral, M. Vicent, etc.). Los fragmentos no constituyen unidad de información y no tienen autonomía para ser analizados o recuperados por separado.

Este trabajo, siendo un primer acercamiento a la extraordinaria casuística de los textos de opinión desde el punto de vista de su recuperación pronominal en bases de datos, será restringido a aquellos textos de los dos primeros tipos que satisfacen de modo más o menos explícito y en mayor o menor grado, la macroestructura: exposición-argumentos-conclusiones. Asimismo, en la observación, son admitidos textos que presentan solo argumentos o solo conclusiones, dando por sentado que el medio/autor ha argumentado o explicitado su posición en textos previos o extraerá conclusiones en textos posteriores. Se remite a investigaciones ulteriores el ajuste o, en su caso, establecimiento de procedimientos derivados de análisis de textos de opinión sui generis, que no presenten estas condiciones dominantes en su macroestructura profunda o cuando ésta sea de tipo fragmentario.

\section{Metodología y aparato teórico conceptual}

Esta investigación se fundamenta en estudios previos realizados sobre géneros periodísticos informativos (García Gutiérrez, 1999 y 2013) abordados de modo mucho más general en otros trabajos centrados en discursos no periodísticos como es el caso de 
la historia colonial portuguesa (Cunha,1990). Pero además del aparato teórico desprendido de ese campo del conocimiento, han sido conceptual y metodológicamente fundamentales la aportación seminal de Charles Fillmore (1968) (teoría de los casos universales), Teun Van-Dijk (2001) (estructuras discursivas), Michel Pêcheux (1978) (análisis automático del discurso), Klaus Krippendorf (2002) (metodología de muestras y análisis de contenido), Santamaría y Casals (2000), Sánchez -Calero et al. (2011) (géneros de opinión y nuevas hibridaciones transgenéricas), y algunas contribuciones clásicas en el campo de la argumentación como las de Chaïm Perelman (1989), de Arthur Schopenhauer (1996) en el área de la "dialéctica erística" y las aportaciones teóricas de Umberto Eco en su concepción de lector-modelo (1993).

Para llevar a cabo la investigación, se parte, por un lado, de una muestra empírica de textos clasificables dentro de los tradicionales géneros de opinión (editorial, análisis, crítica y columna) si bien el criterio de selección dominante reside en que los textos elegidos recojan, en mayor o menor grado y de forma parcial o total, la macroestructura profunda especificada teóricamente en el epígrafe anterior (exposición-argumentos-conclusiones), relegando a estudios posteriores otros géneros o tipologías textuales que, aún pudiendo ser considerados persuasivos o argumentativos, presentaran estructuras o particularidades que no satisfacen tales requisitos. Por otro lado, se formaliza el modo en el que un documentalista de medios simulando a su usuario-modelo -de acuerdo a la concepción de "lector-modelo" de Eco (1993)- debe interrogar el texto para obtener los datos factuales que éste hipotéticamente busca.

En consecuencia, es necesario: 1) la constitución de un corpus de textos de opinión con el fin de determinar si se cumplen las claves macroestructurales indicadas y si es procedente y factible la extracción de información factual por parte del documentalista en un formato de análisis y búsqueda normalizado, 2) elaboración de síntesis estructurales o "macroproposiciones "MPs" a partir de los textos originales con el fin de trabajar sobre "observables" de laboratorio. Como acciones derivadas, pero simultáneas a esta actuación, debe realizarse: a) inventario y depuración de verbos en torno a la acción opinar/analizar a partir de un análisis de diccionarios generales y de sinónimos (Drae, Wordreference y otros), b) inventario y depuración de "conectores" (partículas léxicas que articulan los predicados) procedentes de las MPs obtenidas de la muestra así como de los trabajos de Michel Pêcheux (1978) y de diccionarios de español convencionales y digitales, 3) elaboración de un inventario teórico de categorías de interrogación a partir de un conocimiento empírico de cómo un usuario-modelo (periodistas, reporteros o público general) solicita información factual a un texto de opinión. Tal inventario se obtiene de interrogantes pronominales (Quién opina, Qué opina, Sobre qué opina, Por qué opina...) recogidas de colegas y estudiantes universitarios de periodismo alcanzando rápidamente un umbral de repetición que es cotejado y ampliado tanto con las categorías conceptuales establecidas por la teoría de casos universales como, de modo más próximo, con el repertorio de categorías de análisis y búsqueda ya realizado sobre géneros informativos (12Ws) a fin de contar con un modelo de interrogación pronominal lo más exhaustivo posible (vid 5.2), 4) inserción de las categorías como ángulos de organización de la macroestructura general subyacente en las MPs, 5) asignación de una categoría de análisis y segmentación tex- 
tual a cada modo particular de interrogación pronominal, 6) introducción de protocolos diferenciales en las categorías de interrogación, de acuerdo a las particularidades y matices diferenciales con influencia macroestructural que aportan los textos establecidos como base del estudio, 7) elaboración de MPs exhaustivas en laboratorio conteniendo todas las estructuras de respuestas factibles a categorías de interrogación posibles con el fin de observar los umbrales de rigidez y flexibilidad del procedimiento y proceder, en su caso, a los oportunos reajustes.

Debe hacerse constar que, aunque el procedimiento propuesto es un "automatismo" en el sentido clásico de Pêcheux (1978), no debe colegirse la posibilidad de una inmediata "automatización" o digitalización en todas las fases del proceso. En un ulterior, aunque a medio plazo, desarrollo de este programa de investigación -actualmente en fase lógico-semántica y pragmática de resolución de problemas- sería posible la digitalización del procedimiento a partir de MPs elaboradas por documentalistas con un alto porcentaje de autonomía del software y esporádicas intervenciones humanas, pero la generación automática de MPs a partir de textos de opinión, sin presencia de documentalistas, no es predecible en un futuro razonable.

En todo caso, un software ad hoc liberaría al documentalista de medios de la rutina de la interrogación y representación pronominal una vez elaboradas las MPs (como la indización y extracción de metadatos), haciendo empresarial y económicamente viable la producción de respuestas factuales, en tanto el hecho de continuar "manufacturando" MPs propiciaría la necesidad constante de personal en los servicios de documentación lo que, por otra parte, satisfaría demandas sociales y sindicales además de reorientar las tareas de los documentalistas de medios a un mayor rigor y dedicación a otras funciones como control lingüístico de los fondos o atención a usuarios.

\section{Corpus, observables y construcción de macroproposiciones}

El corpus de trabajo lo constituyen textos de opinión en castellano de medios españoles con el fin de evitar otras legítimas casuísticas idiomáticas que precisan estudios específicos. Dichos medios fueron consultados en las bases de datos MyNews y en versiones digitales de, entre otros, diarios de tirada nacional como El País, El Mundo y $A B C$. A fin de observar la estabilidad macroestructural en el tiempo, se estableció, como segunda condición, que un cincuenta por ciento de los textos procedieran de ejemplares de diarios de finales del siglo XX y, el otro cincuenta por ciento, de textos de finales de la primera década del siglo XXI. La verificación de tal estabilidad macroestructural fue prácticamente inmediata en los primeros textos, en parte debido a la arquitectura canónica apriorística que Santamaría y Casals (2000) aprecian en estos géneros y a la que se aplican los equipos editoriales de los medios escritos convencionales. A pesar de ello, y con el fin de observar posibles derivaciones estructurales $\mathrm{y}$, sobre todo, de realizar un inventario de casos particulares, se decidió dar validez adicional a los estudios teóricos aludidos sometiendo el corpus empírico de textos de opinión, como ya se hizo con otros géneros, a una sencilla técnica de validación basada en Krippendorf (2002).

De acuerdo con ese procedimiento llamado "técnica de la mitad", el corpus inicial se divide en dos mitades y cada mitad debe satisfacer por separado las condiciones y 
objetivos trazados anteriormente. Si en una muestra no se satisface algún requisito, se enriquece con nuevos registros la primera mitad igualándose la otra mitad hasta verse satisfecho (o no, en el caso de negación de la hipótesis). De ese modo, y llegándose a cincuenta textos en cada mitad (centrado en editoriales, análisis, críticas y columnas de las dos épocas recientes aludidas), se observa en primera instancia que todos ellos mantienen los elementos buscados en macroestructuras más o menos explícitas y sometidas a los criterios retóricos y estilísticos de cada medio. Se observa así mismo que, a efectos de analizar, organizar y buscar la información factual que contienen los textos, no son relevantes los elementos diferenciales que desde el punto de vista ideológico y estilístico introducen las distintas cabeceras aunque sí serían decisivos desde la óptica de la representación, dimensión que debe ser abordada en ulteriores investigaciones. En consecuencia, la indagación puede desarrollarse exclusivamente en términos de estructura profunda (macroestructura) sin necesidad de abordar, por el momento, complejas variables temáticas e ideológicas que serán ineludibles en investigaciones posteriores. Finalmente se decide trabajar con síntesis estructurales elaboradas en laboratorio a partir de los textos y no con texto libre, dada la superficie retórica y las propiedades de los géneros de opinión, mucho más heterogéneas que en el caso de los géneros informativos, en los que dieron resultados consistentes.

La construcción de estas síntesis "observables" que, siguiendo a Van-Dijk (2001), denominaremos macroproposiciones, MPs, elimina aún más el riesgo de incidencia estructural de tales elementos temáticos, ideológicos, retóricos o redaccionales propios de cada medio en el procedimiento que se propone. La macroproposición se organizan en función de la macroestructura expuesta (exposición-argumentación-conclusión) pero, a pesar de su proyección de orden semántico -dado que las categorías se "rellenan" con conceptos-, se observa que es posible aislar en cierta medida el significado permaneciendo, también las categorías conceptuales a efectos de este estudio, neutralizadas en cuanto a temáticas o perspectivas ideológicas. Los únicos significados metodológicamente relevantes son los proporcionados por los conectores, pero con el exclusivo fin de entronizar funciones 0 categorías de organización, y el "cambio de temática" dentro de un mismo texto de opinión como indicador de varios subtextos (vid más adelante). A continuación, se presenta el registro general de recogida de datos:

\section{Registro de análisis de géneros de opinión}

\section{Signatura:}

- Descripción externa: autor, título, medio, fecha, página/url

+ Género de opinión: (editorial, análisis, crítica, columna)

- Macroestructura explícita: exposición-argumentos-conclusiones

- Macroproposición:

- Enunciado argumentativo 1: sujeto-verbo-predicado (categorias conceptuales)

- Enunciado conclusivo 2: verbo-predicado (categorias conceptuales)

- Observaciones:

- Conectores en bruto: propuesta de conector normalizado:

- Acciones en bruto: propuesta de acción normalizada: 
La primera variable importante que debe tenerse en cuenta, a partir de la observación de los textos procedentes del corpus, es la presencia de más de un "subtexto" en la unidad de información. Entendemos por subtexto un relato o comentario de cierta suficiencia estructural (esto es, que responda a varias interrogaciones pronominales) e independiente temáticamente del texto inicial (o también subtexto, dependiendo de la profundidad o extensión en relación al segundo subtexto). Obsérvese el servicio esencial que la temática, neutralizada anteriormente, presta en este caso a la partición textual de las MPs. Aunque en el corpus no se registra una aparición constante de dos o más subtextos, estos aparecen con alguna frecuencia y en función de cada cabecera, especialmente en editoriales y análisis según los temas o macrotemas tratados, si bien algunos medios publican un editorial por tema relevante aun en número reducido. Por ejemplo, no es extraño encontrar un editorial que arranca con una crítica al gobierno y termina con comentarios sobre otro tema no subordinado directamente al anterior (por ejemplo, las Olimpiadas). En algunos análisis se ha detectado un macrotema dominante (por ejemplo, la crisis económica) que en realidad se desglosa en varios subtextos (política monetaria, paro, exportaciones y balanza comercial, deuda...) siendo tratado cada uno de ellos con suficiente estructura conceptual y extensión como para otorgarle la consideración de subtexto.

Hay estructura conceptual y extensión suficiente cuando, sobre la base de una temática diferente a la inicial, se observa la existencia de oraciones que aportan datos, valoraciones y acciones dominantes con indicios de autonomía textual, esto es, el subtexto podría ser archivado y recuperado separado de la matriz y ésta mantendría suficiencia estructural y extensión suficientes como para mantener existencia autónoma respecto al subtexto. La decisión sobre el número de subtextos presentes es altamente relevante para la organización categorial de la información en el sistema y una localización más específica y matizada de los datos factuales pero, una vez tomada, el tratamiento de los subtextos se ejecuta en el mismo registro (por ser el mismo autor, tener el mismo titular y formar parte de la misma producción). En consecuencia, un texto que abarca múltiples temas o comentarios sobre personalidades o actuaciones, de modo superficial o enciclopédico, no contará con subtextos. La multiplicidad de temáticas y la decisión de no considerarlas como subtextos no implica un juicio o una minusvaloración del documentalista hacia la producción sino, estrictamente, la determinación de no dividirla en partes (MPs) en atención al usuario-modelo y a la propia lógica macropropositiva general del procedimiento (y no micropropositiva).

En el caso de más de un subtexto, deben construirse tantas macroproposiciones MPs como subtextos detectados (MP1, MP2...) y habrán de ser atribuidas tantas acciones como MPs construidas (a1, a2...). Esta pauta de organización macroproposicional podría ser omitida en los casos en que los diferentes subtextos permitan ser regidos exactamente por la misma acción y que la valoración (véase, más adelante, la dimensión axiológica de la categoría modal) que, en su caso, acompañe a la acción sea idéntica para todos los subtextos. Por ejemplo, si el analista comenta "positivamente" las medidas de política monetaria y las exportaciones en sendos subtextos, estos pueden ser organizados en torno a una misma acción (análisis) con una misma valora- 
ción (positiva). Ahora bien, si el análisis es positivo respecto a esos dos subtextos pero negativo en relación a la política de paro y de deuda -siempre que haya suficiente estructura y extensión- cada valoración debe ser tratada como MPs diferenciadas (una MP que analiza positivamente y otra que lo hace negativamente). En el ejemplo citado podrían construirse dos MPs abarcando, cada una de ellas, dos subtextos.

Otra particularidad de menor frecuencia aparece cuando cada subtexto necesita una acción diferente con independencia de la valoración del autor (analizar, comparar, proponer...), naturalmente mediando estructura y extensión suficientes y, finalmente, algunos textos vienen dotados, por un lado, de suficientes argumentos y, por otro, de suficientes conclusiones, lo que daría lugar a dos enunciados dentro una misma MP (puesto que no ha variación temática para inaugurar una nueva MP).

\subsection{Sobre autores y agentes}

Como antecedentes de esta cuestión, partimos de los citados trabajos sobre noticias y entrevistas, en los que pudo observarse que surgían variables específicas respecto a los agentes responsables de las acciones ejecutadas en los relatos, que hubieron de ser oportunamente atendidas elaborando medidas ad hoc. En el caso de las noticias, el agente del contenido narrado (de existir) forma parte de la narración misma (el atacante en el ataque, los que se reúnen en una reunión, el vendedor en una venta, etc.). Muy por el contrario, tras el análisis de entrevistas, hubo de practicarse una inversión ya que el agente óptimo para el procedimiento de búsqueda no resultó ser el entrevistador sino el entrevistado, quien se convertía en eje de la MP precediendo, en consecuencia, al verbo "declarar". Pues bien, en el caso de los textos de opinión, producciones textuales que giran en torno a derivados de opinar, esto es de una acción dominante extratextual, el objetivo de la búsqueda pronominal de información (mediante simulación de usuario-modelo) exige diferentes convenciones operativas:

- en todos los casos, el nombre del autor del texto de opinión figurará en el correspondiente campo de la descripción externa de acuerdo a las reglas catalográficas que use el medio.

- en el caso de editoriales y, por tanto, de textos "no firmados" que representan la posición del medio, el campo de autor en descripción externa estará, por defecto, vacío (recuérdese que existe un campo de género destinado a informar de la tipología). En este caso, el campo "agente" que forma usualmente parte del análisis mediante categorías pronominales de noticias y entrevistas (como se ha visto, ocupado por "el entrevistado") quedará, asimismo, vacío (y la macroproposición se vertebrara sobre el modo impersonal: se analiza, se opina...).

- en el caso de textos de opinión firmados por periodistas de plantilla, se ubican sus datos como responsables en la descripción externa pero, dado que es su labor habitual, no "forman parte" del comentario ni de lo comentado y, por tanto, el campo destinado a agentes en las categorías de análisis de contenido permanecerá vacío.

- finalmente, en el caso de firmantes que no forman parte del equipo del periódico o que, aún colaborando con asiduidad con el medio, dedican su actividad profesional a otro sector (economistas, novelistas, profesores, políticos...) o son conocidos fundamentalmente por esa otra actividad y no por su vinculación contractual con el medio, 
sus nombres aparecerán tanto en el campo de autor de la descripción externa como en el de agente, dentro del análisis categorial de contenido. Esta excepción se observa dado que, en estos casos, la opinión adquiere relevancia estrictamente en función de la personalidad de quien la expone, un autor externo al medio en cuestión aunque colabore con cierta asiduidad en el mismo, con independencia del valor de sus argumentos (Almodóvar analizando el cine español, Saramago comentando la guerra de Irak, el presidente del gobierno "opinando" sobre su política...).

\subsection{Categorías de interrogación pronominal}

Una vez detectado el número de subtextos relevantes, adjudicada a cada uno de ellos una acción, a cuyo análisis pormenorizado reservaremos el epígrafe siguiente dada su trascendencia para la totalidad del procedimiento (y, por tanto su discusión se omite aquí), y catalogadas las variables de autoría, procede cotejar las categorías teóricas derivadas de la gramática de casos y de las inducciones categoriales procedentes de los textos reales que forman parte del corpus, con los interrogantes pronominales obtenidos de usuarios-modelo (las 12Ws) para desarrollar un primer borrador de categorías empíricas de organización conceptual asociadas a preguntas de orden pronominal que pueden hipotéticamente realizarse a un texto de opinión. Obsérvese que utilizamos provisionalmente el verbo opinar en tanto no se elige un cuasi sinónimo más apropiado en el apartado sobre acciones (vid 5.3):

1. ¿Quién opina? En el caso de personajes (vid 5.1), se obtiene el autor (ag).

2. ¿(Sobre) Qué temas opina? Se relacionan los asuntos relevantes sobre los que se opina, se obtiene el objeto pasivo (op), tantos op como temas analizados, categoría imprescindible en el procedimiento.

3. ¿A/Contra Quién opina? No aplicable. En el caso de un texto de opinión sobre un sujeto individual o colectivo se consideran como asunto o tema, es decir, no "se juzga a X... o se examina a X..." (como haría un juez en su tribunal, un médico en su consulta o un profesor en el aula) sino que se opina sobre X o se analiza X (comportamiento, rendimiento, política llevada a cabo, etc.) por lo que no ha lugar al dativo (además de impedirlo la propia naturaleza de los verbos opinar y analizar en la acepción que nos interesa). Los personajes analizados, por tanto, lo son en tanto roles relevantes de actualidad que se organizan como objetos pasivos (op).

4. ¿Cómo opina? En general favorable o desfavorablemente aunque puede haber matizaciones (urgentemente, comparativamente, preventivamente..., todas ellas insertables como modalidad de opinar), se obtiene el modo (m).

5. ¿A través de Qué opina? Categoría no aplicable ya que en el modelo matriz se reserva a medios físicos o instrumentos a través de los cuales se ejecuta la acción. El medio de comunicación se excluye en este caso pues se hace constar en el campo "medio" de la descripción externa. En el caso de "herramientas" o instrumentos analizados, se trata de objetos pasivos del mismo verbo y no de medios del hecho en sí de analizarlos.

6. ¿Por Qué opina? En el caso de motivo expreso (como portavoz, respuesta o reacción) o contextualizador evidente (proceso electoral, huelga...), se obtiene la causa (c). 
7. ¿Para Qué opina? En el caso de finalidad explícitamente manifestada por el autor (apoyar la retirada de tropas, etc.), se obtiene la finalidad (f). (no aplicable si el fin es obvio o no está explícito).

8. ¿Con Qué consecuencias opina? En el caso de propuestas, resultados o inferencias de los argumentos, es decir, consecuencias del análisis o de la opinión, un segundo e independiente enunciado de conclusiones se muestra más operativo que el mantenimiento de esta categoría conceptual. Por tanto, la consecuencia, como categoría dependiente de la primera acción, no es aplicable, especialmente si se trata de las consecuencias (o reacciones) que el texto provoca puesto que no serían conocidas hasta después de su publicación y, por tanto, no abordables en el texto sino como especulación de su impacto (y, en ese caso, tal especulación sería un asunto tratado por el análisis, es decir, un objeto pasivo).

9. ¿Quién apoya la opinión? No aplicable la categoría asociativa ya que en caso de mencionarse apoyos o antagonismos formarían parte del objeto pasivo (la mención de otros sujetos que opinan como el autor, se considera parte del objeto pasivo)

10. ¿En qué estado opina? No aplicable la categoría situacional ya que en el caso de referirse a su estado (presionado, atacado, censurado, etc.) formaría parte del comentario y, por tanto se trataría también de un objeto pasivo.

11. ¿Cuándo opina? Irrelevante en la mayoría de los casos pues no tiene interés el momento en que se escribió la opinión, salvo raras excepciones que podemos imaginar (relevancia de la fecha en que se hace el comentario). La fecha de publicación, sin embargo, es obligatoria pero pertenece a la descripción externa. Las fechas susceptibles del comentario son objetos pasivos (salvo un aniversario, por ejemplo, que podría ser su causa).

12. ¿Dónde opina? Irrelevante en la mayoría de los casos pues no tiene interés el lugar en el que se escribió la opinión, salvo contadísimas excepciones (relevancia del lugar desde el que se escribe el comentario), aunque a veces acompaña el nombre del firmante ( $\sin$ que signifique conexión entre la ubicación de quien escribe y la opinión o asunto sobre el que escribe). Los posibles lugares sobre los que opina son, en todo caso, objetos pasivos (salvo un traslado del autor a otro lugar, por ejemplo, que podría ser su causa).

\subsection{Acciones}

A diferencia de la naturaleza y relevancia de las múltiples acciones utilizadas en el cuerpo de las noticias (reunir, atacar, secuestrar, vencer, concentrar, juzgar, detener...), en los géneros de opinión, y a efectos de una organización de las categorías conceptuales que permita su interrogación pronominal, las diferentes acciones que proporciona el texto en sí son irrelevantes como ángulo de organización del propio texto, toda vez que son determinadas por una acción verbal dominante (con diferentes matices que veremos) y externa al texto pero de la que dependen todas las demás: se opina sobre el hecho de reunirse, atacar, declarar, juzgar e, incluso, se opina sobre las opiniones de otros (por tanto, como todo hecho sobre el que se opina, se trata de objetos pasivos).

A pesar de que hasta el momento hemos utilizado provisionalmente "opinar" como acción más representativa de los textos de opinión, el cuasi sinónimo "analizar" ha 
mostrado una mayor receptividad (en general, los textos se "prestan" más fácilmente a ser organizados desde "analizar" que desde "opinar", a pesar de que, como se verá, no prescindiremos de "opinar" para ciertos casos) de los textos revisados a la hora de elaborar sus MPs. Para ello, se partió de las acciones componentes del campo semántico de opinar, tanto provistas por diccionarios de sinónimos como al redactar la síntesis de los registros en bruto, configurándose rápidamente un conjunto delimitado y poco extenso de acciones básicas una vez llegados al umbral de repetición proporcionado por las mitades de la muestra, y con un mayor o menor grado de acercamiento al núcleo sémico de "opinar", tales como: analizar, estimar, considerar, exponer, describir, juzgar, valorar, investigar, argumentar, reflexionar, criticar e incluso proponer o concluir. Se eliminan en primera instancia criticar (por confusión con el género crítica), juzgar, valorar e investigar por su exceso de precisión sémica respecto a los fines perseguidos en una acción genérica como opinar. Por su parte, estimar y considerar también son subsumidos, justamente por lo contrario, dada su vaguedad, incluso superior a la de opinar. En cuanto a exponer y describir, se desechan tanto por falta de adecuación como por causar interferencias con acciones propias de los géneros informativos como noticias y reportajes.

Puesto que la elección de una o varias acciones claves de organización textual es pura convención cuando se trata de sinonimia, siendo suficiente una comprobación que deje constancia de la eficacia indistinta de los cuasi sinónimos al ser cotejados con los textos, se observa que en tanto las acciones argumentar y reflexionar pueden ser subsumidas por analizar, deben mantenerse proponer/concluir para dar cobertura a una parte representativa de muchos textos de opinión. En el caso de este par, proponer se subsume en concluir, toda vez que de una conclusión se pueden extraer resultados de argumentos y propuestas de actuación ("se concluye que el gobierno debe incrementar el iva...") pero de un propuesta no se detraen conclusiones o resultados. Resta el problema de la polisemia que conlleva "conclusión" en la representación (como inferencia y como fin de algo), doble sentido que se evita simplemente acudiendo a la regla de número (conclusiones $=$ inferencias, conclusión $=$ fin $)$.

La elección de analizar como acción base del procedimiento obliga, en consecuencia, a la elaboración de dos enunciados en los casos en que los argumentos vayan precedidos o, más habitualmente, sucedidos por conclusiones o propuestas del autor. Obsérvese que se trata de dos enunciados en la misma MP y no de dos subtextos que darían lugar a dos MPs, toda vez que, para alcanzar el estatuto de subtexto, es necesaria una temática diferente algo que no ocurre, naturalmente, en la relación de subordinación que existe entre los argumentos y sus conclusiones. Una estructura macroproposicional de enunciados en torno a las acciones "analizar" y "concluir" se muestra, por tanto, como la más consistente no solo en relación a la organización conceptual de los textos sino especialmente por reflejar de modo más preciso la estrategia discursiva de los géneros persuasivos, esto es, convencer mediante argumentos y propuestas. Por la propia naturaleza y objetivos de los géneros de opinión, puede lógicamente inferirse que conocer mediante interrogaciones pronominales estos argumentos y propuestas de los autores es uno de los objetivos de los usuarios que buscan este tipo de texto periodístico. 
Por otra parte, es necesario que la acción analizar recoja las modalidades y "tonos" del propio texto de opinión, esto es, las estrategias de producción del contenido (crítico, estimativo, orientativo, exhaustivo, comparativo, descriptivo, preventivo...), por lo que debe potenciarse el caso conceptual modal (que también recogería el tono positivo o negativo del análisis).

Veamos los casos verbales sintetizados, ya con una acepción convencionalmente cerrada, estanca e incompatible con otra función en el mismo sistema documental:

- analizar: acción de uso obligatorio para la mayoría de los textos de opinión (editoriales, críticas, columnas y análisis si nos circunscribimos a las acotaciones establecidas) que impliquen una explícita posición favorable o desfavorable del autor (o del medio) basada en argumentos claros y ordenados. En la MP, la acción iniciará un primer enunciado (E1) y se expresará en tercera persona del presente de indicativo sucediendo al sujeto en el caso de firmante excepcional ("Farhadi analiza...") o en impersonal ("Se analiza...") si se trata de un periodista de plantilla.

- concluir: acción que encabeza un segundo enunciado (E2) que sucede al organizado previamente por analizar, incluso en el caso de que la totalidad del texto sean conclusiones o propuestas (por ejemplo, porque el día anterior se han desarrollado los argumentos). Es necesaria la presencia de un enunciado de analizar para contextualizar la macroproposición (y así, generar metadatos del texto en cuestión que lo incardinan a otros previos y afines), pudiendo reducirse al propio verbo analizar acompañado de un objeto pasivo genérico. En cuanto a la sintaxis de la acción concluir: tras el primer enunciado y signo de puntuación, se inserta "Se concluye que" o "Farhadi concluye que..." y, en su caso, las deducciones, resultados o propuestas del texto como objetos pasivos de concluir: MP1-E1: "Farhadi/Se analiza... " MP1-E2: "Farhadi/Se concluye que... op 1. op2. op3....".

- opinar: genérico usado como "comodín" excepcionalmente en el caso de dudas del documentalista acerca del uso adecuado de analizar en textos singulares. También es compatible con concluir en las mismas condiciones mencionadas.

Como se ha expuesto, en ocasiones algunas de las acciones anteriores deben ser matizadas mediante valoraciones (se analiza, se opina... favorable o desfavorablemente o sus sinónimos positiva o negativamente) o especificaciones (se analiza... comparativamente, preventivamente, cautelosamente, estadísticamente...). Los sufijos "-mente" sugieren presencia de modalidad, de ahí que la categoría modal adquiera una relevancia en el tratamiento de los géneros de opinión que no no suele tener en otros géneros. Este punto remite a una investigación auxiliar posterior en el ámbito de la representación, que no puede ser desarrollada aquí dada la limitación de este artículo, con vistas a inventariar, controlar y normalizar todas la modalidades o adverbios que afecten a las categorías verbales seleccionadas.

\subsection{Conectores y ordenación lógico-sintáctica de MPs}

Los sujetos, las acciones o las modalidades establecidas en los epígrafes anteriores desvelan ya una lógica en la combinación y ordenación de todas las categorías conceptuales aplicables a los textos de opinión. Tal organización macroproposicional es imprescindible especialmente con vistas a una futura lectura automática de los regis- 
tros mediante software. En tal estructura, los denominados conectores (Pêcheux, 1978) desempeñan una función organizativa clave. De acuerdo a conjeturas previas confirmadas en el proceso de elaboración de borradores de las MPs, algunas funciones conceptuales vienen ya determinadas por la posición que ocupan en la MP, es decir, que la adjudicación de un conector en esos casos provocaría un forzamiento sintáctico e incluso conceptual: por ejemplo, tras el verbo analizar se ubica un adverbio modal en su caso ("se analiza favorablemente o positivamente...") que no precisa conector explícito pues su inmediata posición posverbal tiene ya función conectiva. Ocurre lo mismo en el caso de no ir seguido de modo sino de objeto pasivo, o incluso de ambos ("Se analiza la política económica..." o "se analiza positivamente la política económica..."). Por otra parte, el análisis puede ir vinculado a causas (motivos inmediatos o contextos) y tener intenciones explícitas (finalidad) en cuyo caso serán necesarios respectivos conectores que, una vez depurados, son "a causa de" y "para que"/"para+infinitivo". En raras ocasiones se observa práctica ausencia de objetos pasivos y, sin embargo, presencia de causa o finalidad (el editorial se elabora como reacción a un problema o para advertir de su existencia y se concluye en la urgencia de una solución, pero no "se analiza" en sí el asunto). En ese caso, la mera exposición del asunto se considera un objeto pasivo y el formalismo sería "acción op, c/f" por ejemplo: "se analiza la amenaza de ataque norteamericano a Siria (asunto, op) a causa del uso de armas químicas (motivo, c) o para advertir del peligro de una guerra total en la región (finalidad, f).

La acción "concluir" solo se acompaña de objetos pasivos, incluso si se advierten modalidades (urgentemente, preventivamente, temporalmente...) éstas serán tratadas como objetos pasivos puesto que afectan a las propuestas o resultados y rara vez a la acción de concluir (podría concluirse "provisionalmente" pero se trata de casuística tan menor que puede ser absorbida por el objeto pasivo evitando la inflación de categorías y campos que rara vez serán satisfechos). El conector entre la acción concluir y cada uno de los objetos pasivos es "que" (se concluye que op1, op2...)

Por su parte, la acción "opinar", relegada a dificultades de uso (por exceso o insuficiencia de su aplicación en ciertos textos) del verbo analizar, necesita un conector explícito (del tipo "sobre") para dar sentido y modularidad a la MP: "se opina sobre la boda del año...". Es recomendable evitar el uso de opinar en favor de analizar cuando sea posible, ya que la unificación verbal propicia una mayor homologación de análisis cuyos rasgos diferenciales se basan en las valoraciones, conclusiones y temas valorados, lo que beneficia la recuperación de estos datos mediante preguntas pronominales al sistema.

\section{Modelo de interrogación pronominal de textos de opinión}

Una vez determinadas las acciones que organizan los tipos de géneros de opinión abordados en la presente investigación, seleccionados y depurados los conectores prácticos y relegadas, subsumidas o eliminadas las categorías conceptuales de menor o nula relevancia al ser aplicadas sobre los géneros que nos ocupan, se deduce el siguiente modelo procedimental ya con el orden lógico-sintáctico convencional que rige las MPs: 


\begin{tabular}{|l|l|l|l|}
\hline Interrogación pronominal & categoría & conector & observaciones \\
\hline MP1 E1 & & & \\
\hline analizar/opinar & & & tercera persona o impersonal \\
\hline ¿Quién analiza? & ag & & sujeto o impersonal \\
\hline ¿Cómo analiza? & $\mathrm{m}$ & -mente & valoración y modos \\
\hline ¿Qué analiza? & op & analizar (vacío) & opinar (sobre) \\
\hline ¿Porqué analiza? & $\mathrm{c}$ & a causa de & \\
\hline ¿Para qué analiza? & $\mathrm{f}$ & $\begin{array}{l}\text { para que, } \\
\text { para+infinitivo }\end{array}$ & \\
\hline ¿Con qué consecuencias analiza? & & & segundo enunciado \\
\hline MP1 E2 & & & \\
\hline concluir & & & tercera persona o impersonal \\
\hline ¿Quién concluye? & ag & & sujeto o impersonal \\
\hline ¿Cómo concluye? & & & no aplicable \\
\hline ¿Qué concluye? & op & que, y que & uno por conclusión adicional \\
\hline ¿Porqué concluye? & & & no aplicable (E1) \\
\hline ¿Para qué concluye? & & & no aplicable \\
\hline Con qué consecuencias concluye? & & & no aplicable \\
\hline
\end{tabular}

Las categorías que en los textos informativos cubren pacientes, instrumentos, consecuencias, tiempo, lugar, e incluso las categorías asociativa y situacional ( $c f r$. Cunha, 1990; autor) no son aplicables en el análisis de textos de opinión, como se ha argumentado en 5.2, dado que se subsumen como objetos del comentario y, por tanto, se incluyen, en su caso, como una enumeración de objetos pasivos (op).

Se observa, en consecuencia, el carácter prioritario de las categorías objeto pasivo y modo en el procedimiento, siendo más secundarias las causas y los fines que han movido a elaborar o persigue el texto de opinión (con un rol más bien contextualizador de la opinión en sí). De hecho, tiene sentido que la mayoría de las demandas pronominales al sistema se centre en los asuntos sobre los que se opina y la valoración emitida por el autor.

Respecto a la acción concluir, no son aplicables las categorías agente, modo, causa y finalidad, toda vez que -de haberlas- serían recogidas previamente por la acción analizar (u opinar) en el primer enunciado por lo que no se requiere duplicación o bien son irrelevantes. Lo que se concluye -el objeto pasivo- sería una categoría de obligada presencia en caso de aparecer esta acción del segundo enunciado.

Veamos, finalmente, un ejemplo de laboratorio en el que se recogen de manera exhaustiva todas las categorías y posibilidades del modelo.

MP E1: "X (ag) (o se) analiza(s) /-/ negativamente (m)/-/ la política comercial española (op1), /particularmente/ /-/ el proteccionismo de Estados Unidos (op2)/y/ las importaciones de materias primas a América Latina (op3) / a causa del/ incremento de la deuda pública (c) /para/ detener el desequilibrio en la balanza de pagos (f).

E2: X (ag) (o se) concluye /que/ el Estado debe revisar su política económica internacional (op1) /y que/ los empresarios deben hacer un esfuerzo para aumentar sus exportaciones a ese continente (op2). 


\section{Conclusiones}

Del material teórico y empírico manejado se extraen las siguientes conclusiones:

- Los textos de opinión presentan, desde la óptica de su gestión documental, una macroestructura constante con parámetros discursivos estables que permiten su protocolización y normalización, con independencia de las temáticas e ideologías de un medio y a pesar de su alto nivel de heterogeneidad temática, estilística y tipológica, en síntesis o macroproposiciones (MPs) que organizan los asuntos, argumentos y propuestas. Tales MPs no solo constituyen un corpus de "observables" sobre los que operar y elaborar un procedimiento de análisis y búsqueda pronominal, sino que en sí mismas son productos documentales y compatibles con otros productos documentales.

- La organización conceptual de las macroproposiciones elaboradas sobre textos de opinión bascula fundamentalmente sobre la acción analizar, y de modo secundario sobre opinar constituyendo un enunciado predicativo. En caso de que el texto de opinión lo requiera, tal enunciado pueden ir acompañado de un segundo enunciado organizado en torno a la acción concluir a efectos de recoger resultados o propuestas de la argumentación.

- La gramática de casos, tras serle practicada una adaptación conforme a las propiedades discursivas de los textos de opinión, se muestra como modelo teórico consistente del que detraer las categorías empíricas de análisis y búsqueda pronominal de estos textos periodísticos atendiendo a su naturaleza específica pero de modo compatible con las extraídas en los géneros informativos, como se ha demostrado en otros estudios. Esta posibilidad favorece un tratamiento diferente, pero bajo formatos de trabajo con campos homologables, para los distintos géneros periodísticos en una misma base de datos integrada.

- La formalización de MPs de textos de opinión, dotadas de conectores explícitos o indicadores posicionales y elaboradas por documentalistas, propicia a corto plazo, mediante la creación de software ad hoc, la automatización plena del resto de tareas documentales de análisis, representación y búsqueda, a partir de las mismas MPs.

- El procedimiento de análisis y búsqueda pronominal propuesto no solo permite la obtención de información factual sino también de otros productos documentales convencionales (referencias, resúmenes, descriptores) simultáneamente derivados.

- Aunque esta investigación ha sido restringida a textos de opinión procedente de prensa española (y en castellano) se estima un ínfimo nivel de dificultad para extrapolar la estructura conceptual propuesta al español de otros países hispanohablantes y de un bajo a moderado índice de complejidad en el caso de las lenguas más próximas derivadas del latín (gallego, catalán y portugués y, con creciente nivel de modificaciones, italiano y francés).

- El procedimiento está concebido para textos de opinión propios del periodismo moderno. En el caso de prensa antigua (para ser aplicado en hemerotecas, por ejemplo), deben ser estudiados cautelosamente los procesos de extrapolación del modelo.

\section{Referencias bibliográficas}

CUNHA, Isabel (1990): Do mito a análise documentária. Sao Paulo, Edusp 
DIJK, Teun A. van (2001): La noticia como discurso. Barcelona, Paidós

ECO, Umberto (1993): Lector in fabula. La cooperación interpretativa en el texto narrativo. Barcelona, Lumen

FILLMORE, Charles J. (1968): "The Case for case", en BACH, Emmon y HARMS, Robert T. (eds.): Universals in Linguistic Theory. New York, Holt Rinehart and Winston, pp. 1-88

GARCÍA GUTIÉRREZ, Antonio (ed., 1999): Introducción a la Documentación informativa y periodistica. Sevilla, Mad

GARCÍA GUTIÉRREZ, Antonio (2013): "Entrevistas periodísticas y textos declarativos: un procedimiento de análisis y recuperación documentales basado en búsqueda pronominal”. El profesional de la información, 2013, julio-agosto, v. 22, n. 4. pp. 315-325

GOMIS, Llorenç (2008): Teoría de los géneros periodísticos. Barcelona, UOC Press

KRIPPENDORF, Klaus. (2002): Metodología de análisis de contenido. Barcelona, Paidós

LATOUR, Bruno y WOOLGAR, Steve (1995): La vida en el laboratorio. La construcción de los hechos científicos. Madrid, Alianza

MARTÍNEZ ALBERTOS, José Luis (2000): Curso general de redacción periodistica. Madrid, Paraninfo

PÊCHEUX, Michel (1978): Hacia el análisis automático del discurso. Madrid, Gredos

PERELMAN, Chaïm y OLBRECHTS-TYTECA, Lucie (1989): Tratado de la argumentación. La nueva retórica. Madrid, Gredos

SÁNCHEZ-CALERO, M ${ }^{\mathrm{a}}$ Luisa et al. (coord. 2011): Géneros y discurso periodístico. Madrid, Fragua

SANTAMARÍA, María Luisa y CASALS, María Jesús (2000): La opinión periodística. Argumentos y géneros para la persuasión. Madrid, Fragua

SCHOPENHAUER, Arthur (1996): El arte de tener razón expuesto en 38 estratagemas. Madrid, Edaf. 